\title{
The Reciprocal Lattice in Hierarchic Quasicrystals
}

\author{
Antony J. Bourdillon \\ UHRL, San Jose, CA, USA \\ Email: bourdillona@sbcglobal.net
}

How to cite this paper: Bourdillon, A.J. (2019) The Reciprocal Lattice in Hierarchic Quasicrystals. Journal of Modern Physics, 10, 624-634.

https://doi.org/10.4236/jmp.2019.106044

Received: April 5, 2019

Accepted: May 18, 2019

Published: May 21, 2019

Copyright $\odot 2019$ by author(s) and Scientific Research Publishing Inc. This work is licensed under the Creative Commons Attribution International License (CC BY 4.0).

http://creativecommons.org/licenses/by/4.0/

\section{cc) (i) Open Access}

\begin{abstract}
Initially, all that was known about diffraction in quasicrystals was its point group symmetry; nothing was known about the mechanism. The structure was more evident, and was called quasiperiodic. From mapping the $M n$ atoms by phase-contrast, optimum-defocus, electron microscopy, the progress towards identifying unit cell, cluster, supercluster and extensive hierarchic structure is evident. The structure is ordered and uniquely icosahedral. From the known structure, we could calculate structure factors. They were all zero. The quasi structure factor is an iterative procedure on the hierarchic structure that correctly calculates diffraction beam intensities in 3-dimensional space. By a creative device, the diffraction is demonstrated to occur off the Bragg condition; the quasi-Bragg condition implies a metric that enables definition and measurement of the lattice constant. The reciprocal lattice is the 3-dimensional diffraction pattern. Typically, it builds on Euclidean axes with coordinates in geometric series, but it also transforms to Cartesian coordinates.
\end{abstract}

\section{Keywords}

Quasicrystal, Icosahedral, Lattice Constant, Reciprocal Lattice, Diffraction, Structure, Metric, Geometric Series, Quasi-Bragg Law, Quasi Structure Factor

\section{Introduction}

Rapidly cooled $\mathrm{Al}_{6} \mathrm{Mn}$ has a phase, supposedly with "Long range orientational order and no translational symmetry" [1]. In fact, it produces sharp icosahedral diffraction owing to hierarchic translational structure. Its five-fold rotation axes are forbidden in classical crystallography owing to space filling constraints, and its icosahedral structure lies outside the limitations of the fourteen Bravais lattices allowed in crystallography. The "long range order" has been long debated, but with minimal progress in understanding how a periodic incident beam, 
whether of electrons or X-rays, diffracts coherently from the "quasiperiodic" lattice.

The foremost beginning lies in the formula: $M n$ has an atomic number that is almost double that of Al. Consequently imaging, in phase-contrast, optimum defocus, transmission, electron microscopy [2], maps the heavier element which has four times the scattering power of the lighter one. Knowledge of the magnifying power of the microscope then reveals that the intervening atoms are $A I$ that forms about the central $M n$ in edge-sharing $A l_{12} M n$ unit cells. The resulting stoichiometry is $\mathrm{Al}_{6} \mathrm{Mn}$, as in the melt before crystallization and as in elemental analysis. Being edge sharing the structure is not space filling. (By contrast, all crystals are both face sharing and space filling.) In icosahedral i- $A l_{6} M n$, the relative atomic sizes are perfect for extremely dense packing in icosahedral coordination [3] [4] [5] [6] $]^{1}$. The unit cells cluster into icosahedral structures in at least four tiers of icosahedral hierarchy, seen in the image. There are good reasons for projecting the hierarchy to extend infinitely.

The stereogram of the principal axes and of principal reflecting planes in the icosahedral structure is, in its simplest form, 3-dimensional; indexed in geometric series; and complete [7]. Axial diffraction patterns have been likewise indexed [3] [4]. Every component in every beam, in each diffraction plane normal to major axes, has been precisely indexed by additions of one or two members of the geometric series. With this description of the pattern, and knowing the structure, the diffraction is simulated by normal scientific method [8] [9] $]^{2}$, as outlined below.

Because Bragg diffraction is well understood in its wide application to crystals, it is a mildly useful foil for understanding diffraction in quasicrystals, but only if differences are emphasized. Bragg's law describes the linear series of diffraction orders, $n=0,1,2,3 \ldots$, that result from periodically spaced reflecting planes of atoms. For the simple case of high energy electron diffraction from a cubic crystal, we can write approximately:

$$
n \approx \frac{d \cdot \Theta}{\lambda}=\frac{a \cdot \Theta_{h k l}}{\lambda\left(h^{2}+k^{2}+l^{2}\right)^{1 / 2}}
$$

where the scattering angle, $\Theta \approx 2 \sin (\theta)$ for the Bragg complement to the angle of incidence $\theta$, while $d$ is the periodic interplanar spacing; and $\lambda \ll d$ is the wavelength of the incident beam. For each indexed scattering angle $\Theta_{h k l}$ in a cubic crystal with lattice parameter $a$, there corresponds an interplanar spacing $d_{h k l}=$ $a /\left(h^{2}+k^{2}+\mathcal{P}\right)^{1 / 2}, h, k$, and $l$ positive integral.

By contrast, the quasicrystal (QC) does not have regular, periodic, interplanar spacings, and the orders in the diffraction pattern are in geometric series $\tau^{m}$. Moreover, the relations between scattering angle $\Theta, d^{\prime}$ and $\lambda$ were a priori un${ }^{1}$ All diatomic quasicrystals have diameter ratios for central atom/matrix atoms of $(1+\tau)^{1 / 2}-1$, i.e. equal to the diagonal/side ratio in the golden rectangle.

${ }^{2}$ By an application of Ockham's razor, "Dimensions should not be multiplied without necessity": because axioms are unquestioned in math, they are meaningless in physics-as are all other unfalsifiable hypotheses [8] [9]. 
known. Supposing modified relations, the following solution is consistent with the diffraction pattern:

$$
\tau^{m} \approx \frac{d^{\prime} \Theta^{\prime}}{\lambda}, m \text { positive or negative integral, }
$$

with details to be determined by simulation and experimental consistency. Every atom scatters. We have to suppose that in any given QC orientation, either most atoms scatter randomly but that atoms in adjacent planes may filter coherent scattering for the appropriate $d^{\prime}$ spacing; or that all atoms in the geometric structure coherently scatter the incident periodic wave. The former supposition is falsified because known interplanar spacings do not match Equation (1); the following evaluation of quasi scattering factors is a numerical solution that describes the coherent sharp diffraction that follows Equation (2). Complete analysis is work in progress.

Meanwhile, consistency is required between structure, scattering, and measurement. In particular, and consequent on optimum defocus imaging, it is clear that the unit cell has dimensions $a(1 \times \tau)$, where $a$ is the quasilattice parameter that requires measurement, and the bracket gives the dimensions of the golden rectangle [3] [10]. (All 30 edges in the icosahedron belong to 15 golden rectangles through its center.) Given the structure, the parameter must be close to the diameter of the $\mathrm{Al}$ atom. Neighboring cells have common edges, but not common faces.

In crystals, Equation (1) provides the principal condition required for diffraction, and it is harmonic in order $n$. However, the equation is not a sufficient condition. Many indexed beams that are allowed by the equation are in fact forbidden by symmetric details within the periodic unit cells [11]. Classically, the structure factor method is used to identify them. By contrast, the quasicrystal unit cell is not periodic, so a more complex calculation is needed to identify and simulate the many and varied, beam intensities that occur in the geometric series diffraction pattern. Knowing, from optimum defocus imaging, the detailed structure of i- $A l_{6} \mathrm{Mn}$, we can proceed to compare diffracted beam intensities by simulations of Quasi Structure Factors. We need to simulate, not only the supposition of Equation (2), but also to measure the lattice parameter a, along with beam intensities and other features of the diffraction pattern. The fact that the known structure-at least when considered as ideal and defect free-is uniquely icosahedral, is confirmation of its correspondence with the point group symmetry of the diffraction pattern. The quasi structure factors are calculated from the known ideal structure.

\section{Hierarchic Icosahedral Structure}

Figure 1 shows a foil section of $\mathrm{i}-A \mathrm{I}_{6} M n$ imaged in phase-contrast, optimum-defocus, electron microscopy [3]. White dots map $M n$ because of its higher atomic number, i.e. in the thin foil, the principal contrast is due to the greater scattering power of $M n$ (atomic number 25) compared to $A l$ (atomic number 13). 

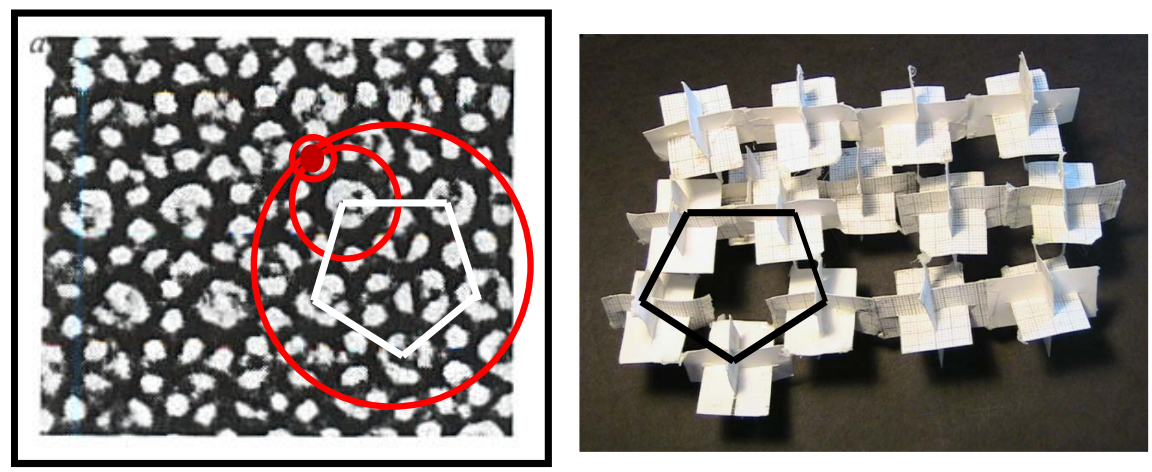

Figure 1. At left, phase-contrast, optimum defocus electron micrograph ([3] pp. 66-67) of $i-A l_{6} M n$ reveals icosahedrally coordinated $M n$ atoms as white dots and the filled red circle. The red circles outline 4 tiers of ordered icosahedral clusters. The large red circle outlines a supercluster section containing cluster centers at the corners of the white pentagon. At right, the black pentagon models the section by mirror image. Here, the triads of golden rectangles represent icosahedral clusters of twelve unit cells (two on each central axis), icosahedrally coordinated.

The $M n$ atom is the first of four tiers of icosahedral structure (red circles). When combined with all of the information given by the point group symmetry of the diffraction pattern [1]; and with the 3-dimensional stereogram of principal axes and diffraction planes [7]; while furthermore having patterns in geometric series completely indexed, the structure is evident. This series is intuitively consistent with the idea of hierarchic structure, and is proved to be so by calculations of quasi structure factors. While the model is an ideal representation for the rapidly quenched intermetallic alloy; the probability of deriving this image from any other structure that has been proposed (e.g. [12]) is absurdly small.

\section{Quasi Structure Factors (QSF)}

Whereas, in crystals, the Bragg condition (Equation (1)) determines harmonic factors $n$ that relate $\lambda$ to $\Theta$; corresponding structure factors sum atomic scattering amplitudes that determine intensities and forbidden lines. Likewise in quasicrystals, the dual importance of the quasi-Bragg condition and quasi-structure factors applies, but especially so because line intensities are many and varied. The general application of the formulae has been previously described [5], but we need to revisit the prerequisite of indexation. Begin with the atomic coordinates for the unit cell and cluster:

Unit cell $\left(r_{u}\right): M n:(0,0,0)$

$$
A l: 1 / 2( \pm \tau, 0, \pm ; 1), 1 / 2(0, \pm 1, \pm \tau) \text {, and } 1 / 2( \pm 1, \pm \tau, 0)
$$

Cell or Cluster centers $\left(r_{c c}\right): 1 / 2\left( \pm \tau^{2}, 0, \pm \tau\right), 1 / 2\left(0, \pm \tau, \pm \tau^{2}\right), 1 / 2\left( \pm \tau, \pm \tau^{2}, 0\right)$.

In crystals (that obey Equation (1)), the structure factor formula projects each atomic site at vector $r_{i}$ in a unit cell onto a selected plane normal having integral indices $h k l$ (Equation (1) with $c_{s}=1$ ). By summing the projected cosines on non-equivalent atoms, an amplitude is obtained that corresponds to the intensity 
of the ( $h k l$ ) diffracted beam after a crystal is oriented to the Bragg condition. For example, the closest crystalline approximant to $\mathrm{i}-\mathrm{Al}_{6} \mathrm{Mn}$ is second phase, face centered cubic (fcc) $A l_{x} M n, x>>6$ [7]. About the [110] axis diffracted beams have either all indices even or all odd; otherwise diffraction is forbidden by the symmetry of the unit cell [11]. The structure factors $F_{h k l}$ have values equal to either 0 or 4 . The atomic scattering factor is $f_{i}$ and, in the crystal, the metric $\mathrm{c}_{s}=1$ :

$$
F_{h k l}=\sum_{i=1}^{N} f_{i} \cos \left(2 \pi \cdot c_{s}\left(\overline{h_{h k l}} \cdot \overline{r_{i}}\right)\right)
$$

By contrast, in icosahedral quasicrystals (that obey Equation (2)), quasi-structure factors (QSFs) are more complex and more varied: because it is not structurally periodic, the summation in Equation (4) is extended to clusters and superclusters indefinitely. Write the vector from the origin to each atom in a cluster $\overline{r_{c l}}$ as the sum of a unit cell vector $\overline{r_{u}}$ used previously, with a vector to the cell centers in the cluster $\overline{r_{c c}}: \overline{r_{c l}}=\overline{r_{c c}}+\overline{r_{u}}$. Then since

$$
\sum_{i}^{N \text { cluster }} \exp \left(\overline{h_{h k l}} \cdot \overline{r_{c l}}\right)=\sum_{i}^{12} \exp \left(\overline{h_{h k l}} \cdot \overline{r_{c c}}\right) \times \sum_{i}^{13} \exp \left(\overline{h_{h k l}} \cdot \overline{r_{u}}\right)
$$

with corresponding summations over cell centers and unit cell sites, $N_{\text {cluster }}=$ $N_{c c} \cdot N_{u}$ the QSF for the cluster may be calculated:

$$
F_{h k l}^{\text {cluster }}=\sum_{i=1}^{12} \cos \left(2 \pi \cdot c_{s}\left(\overline{h_{h k l}} \cdot \overline{r_{c c}}\right)\right) \cdot F_{h k l}^{\text {cell }}
$$

and the calculation iterates on superclusters orders $1,2,3 \ldots p$, by inclusion of the stretching factor $\tau^{2 P}$ :

$$
F_{h k l}^{p}=\sum_{i=1}^{12} \cos \left(2 \pi \cdot c_{s}\left(\overline{h_{h k l}} \cdot \tau^{2 p} \overline{r_{c c}}\right)\right) \times F_{h k l}^{p-1}
$$

$F_{h k l}^{\text {cluster }}$ may also be written $F_{h k l}^{0}$. The iteration is important when the calculation is performed over large clusters, for then truncation errors due to the many additions tend to randomize answers. Though large clusters yield precise conclusions [3] [4] here we will be concerned rather with diffraction effects at the cell and cluster levels up to supercluster order 2. Notice, by contrast with (periodic) crystals, that a line that is forbidden, or partly forbidden at the cell level may be allowed by different greater dimensions at cluster and higher order levels. We therefore begin by supposing that the diffraction is in first Bragg order $n=1$, and proceed to determine details in the quasi Bragg law (Equation (2)), including the calculated ratio $d h / d c_{s}$. The negative derivative that will be calculated is used to refine former results.

Figure 2 shows simulations for QSFs in the geometric series (100), ( $\tau 00)$, ( $\left.\tau^{2} 00\right),\left(\tau^{3} 00\right)$ and $\left(\tau^{4} 00\right)$, all plotted against varying values for the metric $c_{s^{*}}$ Each plot contains a single peak centered at $c_{s}=0.894$; no other significant structure is found between $1.2>c_{s}>0.8$. The plots confirm the geometric series diffraction that is observed in diffraction patterns, and that was discovered in the stereograms [7] and indexation [3]. Moreover, the plots confirm the explanation for the geometric series being due to the hierarchic structure. The series shown is 


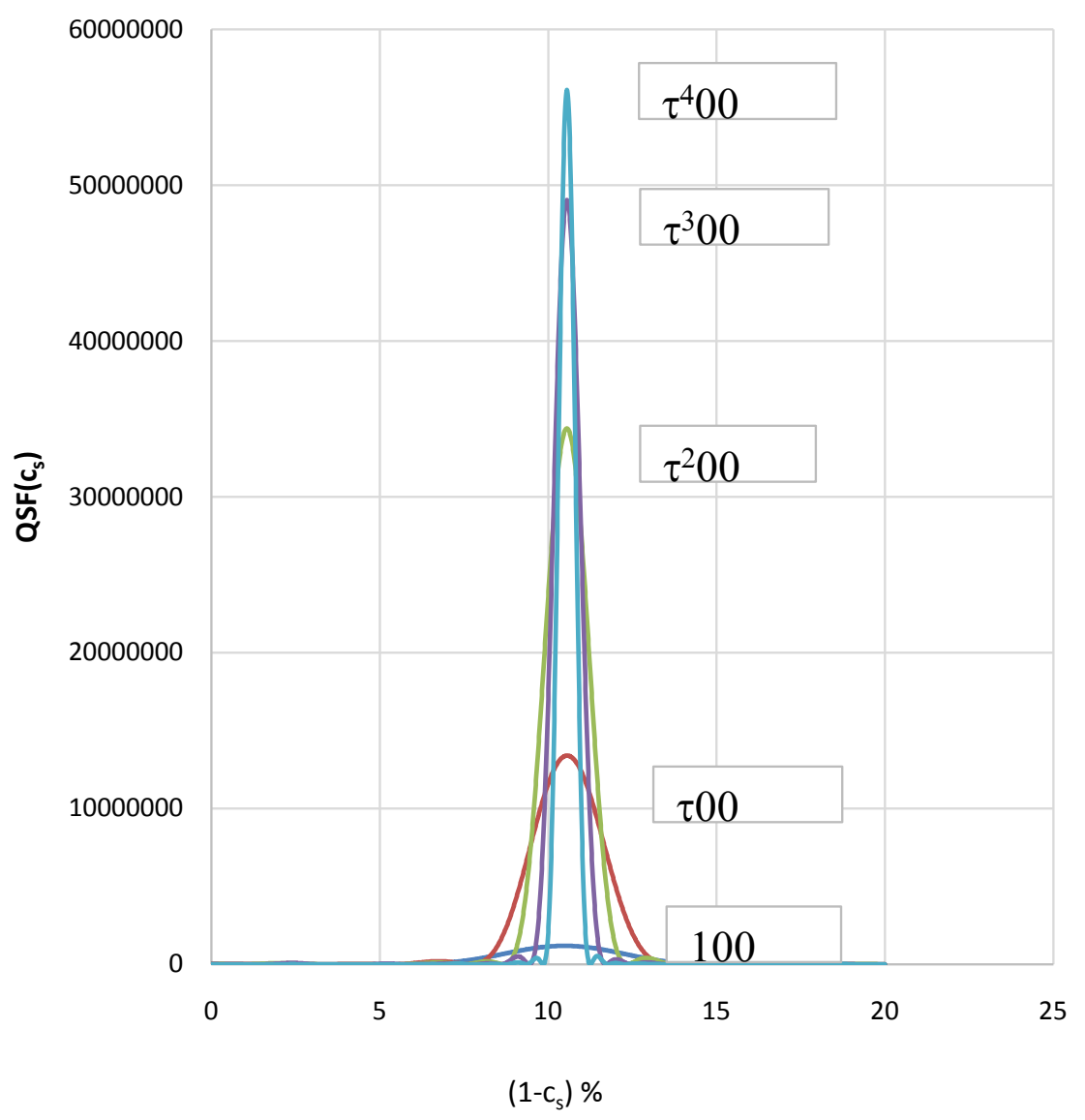

Figure 2. QSFs simulated for indexed lines as shown from an i- $A l_{6} M n$ supercluster order 2. The lines peak consistently when $\mathrm{c}_{s}=0.894$ with similar intensities (FWHM). By contrast corresponding QSFs for Bragg conditions $\left(c_{s}=1\right.$ and $\left.n=0,1,2,3 \ldots, m=0\right)$ are unregulated, with more or less random QSF structures, spread out and very weak, like noise.

illustrative: all simulated peaks from the quasicrystal occur with the same metric (i.e. the value of $c_{s}$ when the QSF peaks). The plots also emphasize the critical and creative importance of the metric for the confirmation of Equation (2).

The significance of Figure 2 is further illustrated by comparing equivalent scans calculated for crystals [4]. Owing to the condition expressed in Equation (1), all "QSFs" calculated for crystals peak only on the ordinate axis $\left(c_{s}=1\right)$. Moreover, in the simple case of fcc $A l$ for example, the number of values calculated is limited, typically 4 or 2 or zero, there being only four non-equivalent atoms in the unit cell. By contrast, not only do quasicrystal QSFs peak only at a precise off-Bragg condition $\left(c_{s}=0.894 ; \Theta^{\prime}=\Theta / c_{s} ; d^{\prime}=d \cdot c_{s}\right)$ in Equation (2) and Table 1 below, but the intensities have a wide spectrum of values over various diffraction beams $(h k l)$. This variety is due to the larger number of atoms that contribute to the diffraction from large clusters, i.e. much greater than the number in a single unit cell.

The peaks describe a new physical effect in quasicrystals. In crystals, Bragg's law (as in Equation (1)) supposed a simple model, with elementary mathematics and clear experimental evidence. By contrast, quasicrystals are described by 
Table 1. Calculation for the lattice parameter $a$ in the quasicrystal from measurements made by assuming Bragg diffraction.

\begin{tabular}{|c|c|c|}
\hline Bragg condition & Quasi-Bragg condition & Comment \\
\hline$n \lambda \approx d \Theta$ & $\tau^{m} \lambda \approx d^{\prime} \Theta^{\prime}$ & $\begin{array}{c}\text { Including metric, ('), } n, m \text { integral } \\
m=- \text { infinity, }-1,01,2,3 \ldots \text { or } \\
h^{\prime}=0, \tau^{-1}, 1, \tau, \tau^{-1}, \tau^{-2}, \tau^{-3} \ldots\end{array}$ \\
\hline$d=a / h$ & $d^{\prime}=a^{\prime} / h^{\prime}$ & $r$ is fixed within known models \\
\hline & & $\begin{array}{l}\text { Diffraction depends on harmonic } r . h \text { in SF } \\
\text { for Equation (1) \& QSF for (2) }\end{array}$ \\
\hline$c_{s}=1$ & $c_{s}<1$ & $\begin{aligned} & \text { In quasi Bragg } h^{\prime} c_{s}<h \\
d_{\tau 00}^{\prime} & =0.205 \times c_{s} \mathrm{~nm} \\
d_{100}^{\prime} & =0.205 \times c_{s} \times \tau=0.296 \mathrm{~nm} \\
& =a^{\prime}\end{aligned}$ \\
\hline
\end{tabular}

equally simple mathematics (Equation (2), Equations (5)-(7)), and equally clear diffraction evidence, but require a more complex model. The equations and evidence imply that the sums on the cosines in Equation (7), when each is multiplied by planar densities taken from the structure, result in specular reflection from imaginary planes that are $10 \%$ shifted from the corresponding Bragg condition. This is the most remarkable feature of the hierarchic scattering that is observed in geometric series (Equation (2)). The shift is $1-c_{s}$ at the peak.

Notice that in the experimental high energy electron diffraction pattern, the intensities of the higher orders are further and normally reduced by the deviation parameter, that result from larger scattering angles [12]. The "third bright ring" is exceptional and is described further below where it is used to define the quasi lattice parameter.

All atom sites in Equation (7) were summed for the supercluster order 2, but with four adjustments: edge cell atoms that were counted twice were entered by halving the atomic scattering factor $f_{A}$ s secondly, those that were counted thrice, at intersections of three cells, were entered with one third $f_{A l}$. Thirdly, mobile sites, where one atom shares two sites were counted as for edge sharing; and fourthly, some details were ignored because of their small effects and uncertainty. For example, Pauling's observation [13] that though icosahedral structures are known in crystals having large unit cells, the structures contain holes at their centers. These holes contain less than $2 \%$ of the volume of icosahedral structures. In principle the holes can be filled with octahedral structures (the octahedron is a subgroup of the icosahedral group and so should not display obvious effects in diffraction). However, we have chosen to ignore hole fillings because various fillings are possible.

Given the method of indexation described, the scattering is thus seen to be coherent when the metric $c_{s}=0.894^{3}$. Figure 2 is an example of the general re${ }^{3}$ When the calculations were undertaken earlier under the assumption of second Bragg order, $n=2$, a different value was found with a value for $1-c_{s}$ about $5 \%$ instead of $10 \%$ as in Figure 1 . On reflection, this will be seen to be consistent; and the assumption depended on the symmetry of the unit cell. However, since it is now obvious from Equations (5)-(7) that structure factors depend on both the unit cell and the on the clusters, the earlier hypothesis that $n=2$ is corrected and is replaced by indexation based on $n=1$. 
sult that is calculated in quasicrystal diffraction. We now need to consider how to apply the calculated metric for the hierarchic structure.

Figure 3 illustrates five simulations using four imagined pseudo-indices having $h=1.5,1.6,1.7$ and 1.8, and also the midway index at $h=1.618034 \ldots$, the ( $\tau$, $0,0)$, i.e. consistent with quasi-Bragg diffraction in Equation (2), with $m=1$. The pseudo-indices are anharmonic and unreal because the corresponding $m$ is fractional. The central peak in Figure 3 is the second peak in Figure 2. The peak intensities are constant. By plotting, separately, the index against the peak positions, the linear slope $d h / d c_{s}$ is found to be -0.186 , i.e. negative. A similar relationship between indexation with interplanar spacing applies to quasicrystals as to crystals (namely $d_{h k l}=a /\left(h^{2}+k^{2}+P\right)$ ) so the derivative $d d_{h k l} / d c_{s}$ must be positive. This fact is used to derive the true quasicrystal lattice parameter $a^{\prime}$ in Table 1. This parameter is the corrected value that, previously, was supposedly measured by incorrectly by assuming second order $(n=2)$ Bragg diffraction in the quasicrystal. The calculated slope demonstrates the fact that the lattice parameter that was previously measured by assuming Bragg diffraction, was overestimated (Table 1).

\section{Quasi Lattice Parameter and Reciprocal Lattice}

The parameter is at first sight ambiguous because the unit cells are edge sharing. Fixed are the stretching factor $\tau^{2}$; the icosahedron cell length $\tau \cdot a$, and its side length $1 \cdot a$; but the two most obvious subordinate cells are overlapping cubes side $\tau \cdot a^{\prime}$, or floating cubes side $a^{\prime}$ with severe underfilling. The measured lattice parameter is reported to be $a=0.205 \mathrm{~nm}$ [12] [14], i.e. measured from the "third bright ring" [1] [15], following the methods of classical Bragg diffraction. In the crystallography of cubic materials, an interplanar spacing $d$ and scttering vector $\Theta$ depend on $h^{-1}$ and $h$ respectively, in a (h00) line. However in the quasicrystal,

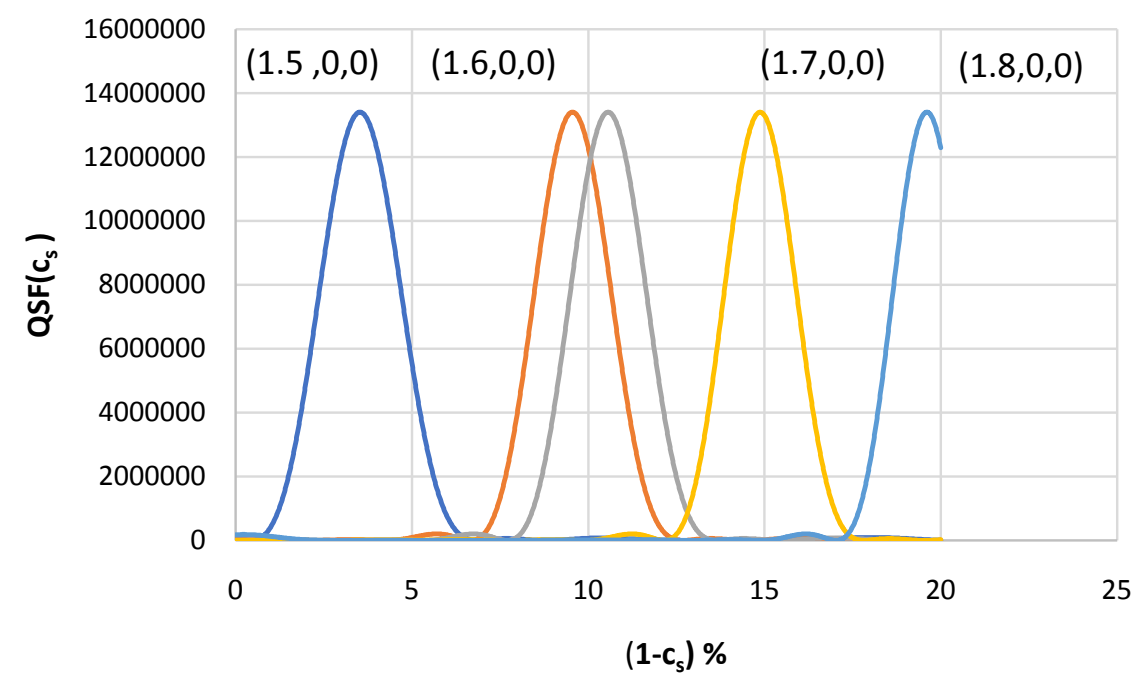

Figure 3. Simulations for QSFs plotted against various trials of metric $c_{s}$, where the index is also varied by imaginative construction. The relationship is almost linear $d h / d c_{s}=$ -1.86 . 
the off-Bragg condition requires correction for $c_{s}$. Then the cell side length measures as $0.205 \times c_{s} \times \tau=0.296 \mathrm{~nm}$ (Table 1), or $3 \%$ greater than the diameter of $A l$ in the pure metal. The measurement of $a^{\prime}$ depends on correct conventions of indexation and definition. It is convenient to define the lattice parameter $a^{\prime}$ in i- $A l_{6} M n$, as the normal diameter of $A l$ in the unit cell of pure $A l$. We therefore define the quasi-lattice parameter as the width of the icosahedral unit cell. When we progress to the reciprocal lattice, it is convenient to express the parameter in simplified icosahedral units, $a^{\prime}=1$. This corresponds with the fact that in hierarchic quasicrystals, diffraction measurement occurs through the compromise multi-spacing effect, or metric, $c_{s}$.

Quasicrystal crystallographers sometimes ask about the reciprocal lattice in quasicrystals because the reciprocals provide important understanding in measurement from crystals.

This understanding extends to most of the solid state physics of crystals, including energy band structures, conductivity etc. In early researches, alternative bases were sometimes used to index the diffraction pattern, but the Euclidean axes of the $O_{h}$ subgroup of the icosahedral point group are now standard.

Should we use coordinates that are Cartesian, or coordinates in geometric series? The former represent the unit cell parameter $a$ in linear order, and so are the more convenient choice for some purposes. More generally, the geometric series expansion of the hierarchic solid relates to the true structure.

Meanwhile, the reciprocal lattice in momentum space has the same point group symmetry as the solid structure and Euclidean axes are again the best choice. The 3-dimensional lattice may be calculated from QSFs using power values for $h, k$ and $l$ equal to $\tau^{m}, m=-$ infinity, $-1,01,2,3 \ldots$ A diffraction pattern is a projection of a reciprocal lattice onto two dimensions. The reciprocal lattice may be recorded by abscissae coordinates in either linear or geometric series. The latter series has been used to construct dispersion curves ([16] [17] p. $17)$, with energy space ordinates plotted against $k$ vector abscissae. In geometric coordinates, the free electron dispersion is then a straight line with slope 2 . Meanwhile, because the geometric series, base $\tau$, is a particular Fibonacci sequence, quasi-Bragg reflected beams in the extended zone scheme have the slope -1 . Zone boundaries are constructed on linear units $m$ in the power series $\tau^{m}$ coordinates. By simple transformation, extended zones can be represented on alternative linear coordinates. Another example is for high energy electrons used in imaging. Dispersion is represented in momentum ordinates with scattering vector abscissae ([17] p. 51). This dispersion is useful for describing the dynamics of quasi-Bloch waves [5]. Each representation, geometric series or linear, has its merits, while results are mutually transformable.

Knowing the clear icosahedral structure, corresponding progress is also expected in defect structures (e.g. [7]) in rapidly quenched material. A further expectation is application of the quasi structure factor method to 2-dimensional quasi-crystalloid structures, i.e. with periodicity on the c-axis but having 10-fold, 6-fold or 8-fold rotational symmetries (e.g. [17] p. 68). We use math, but only 
when it is falsifiable (cf. [4] p. 83); and not where it is falsified.

\section{Conclusion}

We do not ask "What is a quasicrystal?" [18]. "The short answer is no one is sure." With the mapping of $M n$, and with the extension of the hierarchic structures from cell to cluster to superclusters of infinite order, the structure is known. With the stereographic projections of principal axes and diffraction planes indexed in three dimensions; with complete indexations based on summations of backbone geometric series in 3-dimensions; with the representation of all diffraction beams by quasi structure factors, the diffraction pattern is matched to the known structure. It is no wonder that the matching lattice constant and reciprocal lattice are consistently measured; the wonder is that periodic probes, whether of X-ray or electron beams, scatter coherently from the hierarchic, quasiperiodic structure. This coherence is firmly demonstrated by numerical methods applied on an off-Bragg condition, as this paper emphasizes. Though the quasicrystal lattice is geometric, the reciprocal lattice is easily transformed to linear coordinates.

\section{Conflicts of Interest}

The author declares no conflicts of interest regarding the publication of this paper.

\section{References}

[1] Shechtman, D., Blech, I., Gratias, D. and Cahn, J.W. (1984) Physical Review Letters, 53, 1951-1953. https://doi.org/10.1103/PhysRevLett.53.1951

[2] Bursill, L.A. and Peng, J.L. (1985) Nature, 316, 50-51. https://doi.org/10.1038/316050a0

[3] Bourdillon, A.J. (2009) Quasicrystals and Quasi Drivers. UHRL, San Jose.

[4] Bourdillon, A.J. (2012) Metric, Myth and Quasicrystals. UHRL, San Jose.

[5] Bourdillon, A.J. (2016) Journal of Modern Physics, 7 43-50. https://doi.org/10.4236/jmp.2016.71005

[6] Bourdillon, A.J. (2011) Logarithmically Periodic Solids. Nova Science, New York.

[7] Bourdillon, A.J. (2013) Micron, 51, 21-25. https://doi.org/10.1016/j.micron.2013.06.004

[8] Popper, K.R. (1959) The Logic of Scientific Discovery. Hutchinson, London. https://doi.org/10.1063/1.3060577

[9] Popper, K.R. (1982) Quantum Theory and the Schism in Physics. Hutchinson, London.

[10] Huntley, H.E. (1970) The Divine Proportion. Dover, New York.

[11] Cullity, B.D. (1978) Elements of X-Ray Diffraction. Addison-Wesley, Addison.

[12] Tsai, A.P. (2008) Science and Technology of Advanced Materials, 9, 1-20.

[13] Hirsch, P., Howie, A., Nicholson, R.B., Pashley, D.W. and Whelan, M.J. (1977) Electron Microscopy of Thin Crystals. Krieger, New York.

[14] Bourdillon, A.J. (1987) Philosophical Magazine Letters, 55, 21-26. 
[15] Pauling, L. (1985) Letters to Nature, 317, 512-514. https://doi.org/10.1038/317512a0

[16] Bourdillon, A.J. (2009) Solid State Communications, 149, 1221-1225.

[17] Bourdillon, A.J. (2010) Quasicrystal's 2D Tile in 3D Superclusters. UHRL, San Jose.

[18] Senechal, M. (2006) Notices to the American Mathematical Society, 3, 886-887. 